\section{WOMAN WITH RELAPSING FEVERS}

\author{
Mary Kate McCullen, $M D$
}

\section{Case Presentation}

A 50 year-old Nigerian woman with a past medical history significant for type II diabetes and hypertension presented to the ED with a chief complaint of recurrent fevers and chills. The patient was in her usual state of health until approximately 6 weeks prior to admission, while during a visit with family in Nigeria she noted the onset of high fevers and general malaise. Initially, her fevers occurred daily for a period of one week, and were associated with chills, fatigue, loss of appetite, and myalgias. During this time, she was not evaluated by a physician, nor did she take any medication. Her symptoms seemed to resolve however, and she felt reasonably well. She then returned home to the United States, approximately 4 weeks prior to admission. Upon return, she again experienced one week of recurring fevers/chills, as well as the above generalized symptoms. With no evaluation or intervention, her symptoms improved. Two weeks later, the same pattern of symptoms returned and the patient reported to the ED.

Upon presentation, the patient complained of subjective high fevers, shaking chills, anorexia, fatigue, generalized weakness, myalgias, and 15 pound weight loss during the prior 6 weeks. She denied sick contacts and known TB exposures. She traveled with her son, who remained in his usual state of health. She reported that the areas to which she traveled were heavily mosquito-infested.

Her past medical history was significant for type II diabetes and hypertension. Her home medications included metformin 500 mg twice daily and diovan/HCTZ 160/25 once daily. There were no recent medication changes and she denied drug allergies. She had no history of alcohol, tobacco, or substance abuse. Her mother and father were both alive and well, living in Nigeria. She is employed as a chef, but had not been to work since before her visit to Nigeria.

Physical exam revealed a well-nourished woman in no apparent distress. Her temperature was 102.8; pulse 88 beats/minute; blood pressure $144 / 78 \mathrm{~mm} \mathrm{Hg}$; respiratory rate 16 breaths/minute; and pulse ox $100 \%$ on room air. Her sclera were anicteric and mucous membranes were dry. She had a normal S1S2, with a regular rate and rhythm, and no murmurs, rubs, or gallops. Her lungs were clear to auscultation bilaterally and she had no peripheral edema. Her abdomen was soft, non-tender, non-distended, with no hepatosplenomegaly. Her neurologic exam was non-focal; she was awake, alert, and oriented to person, place, and time.

Laboratory evaluation showed a normal complete blood count, chemistry panel, and liver function panel. A urinalysis revealed trace protein and trace ketones. Her EKG showed NSR with no ischemic changes. A blood smear was reviewed and revealed intraerythrocytic ringforms consistent with Plasmodium (Figures 1 and 2). A diagnosis of malaria was made and the patient was immediately started on quinine and doxycycline. Despite oral therapy, the patient was admitted to the hospital to follow her fever curve and await speciation. The patient remained afebrile after -36 hours of antibiotic therapy. The microbiology lab

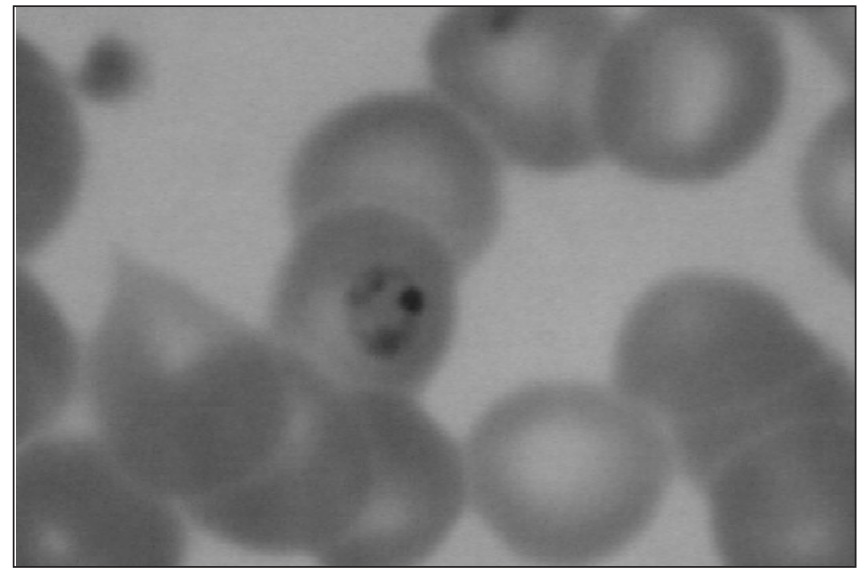

Figure 1.

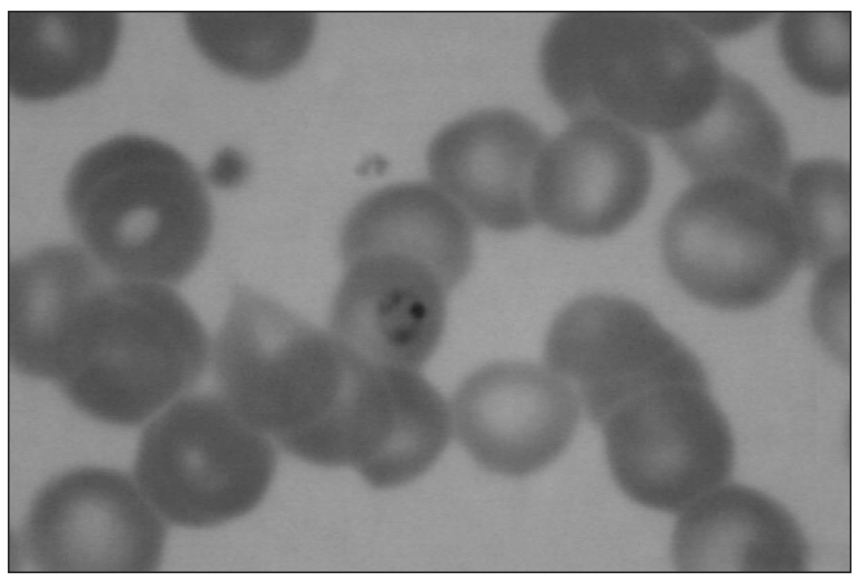

Figure 2.

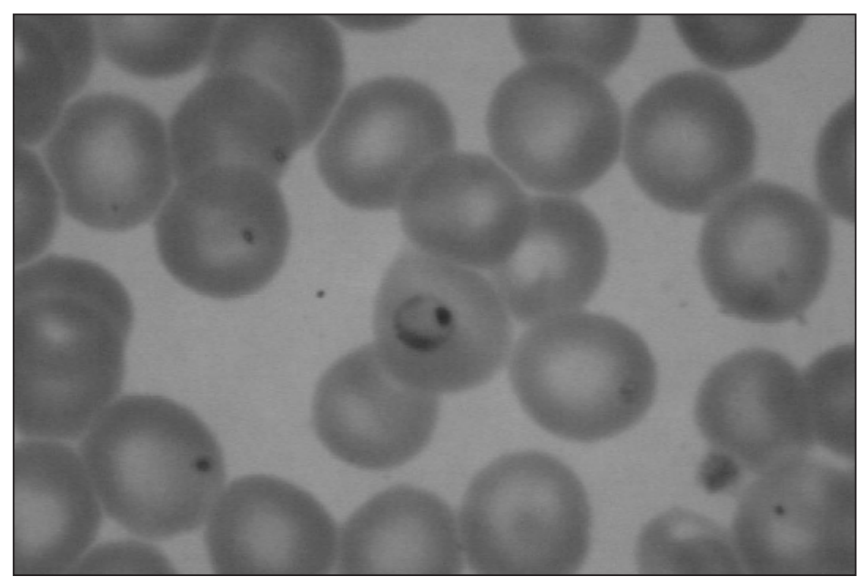

Figure 3.

performed a Giemsa stain of thin and thick smears, revealing Plasmodium falciparum with a $20 \%$ parasitemia level. Her labs were followed in house as well, with no evidence of renal compromise or LFT abnormalities. She was discharged home to complete a 7 day course of quinine and doxycycline. 


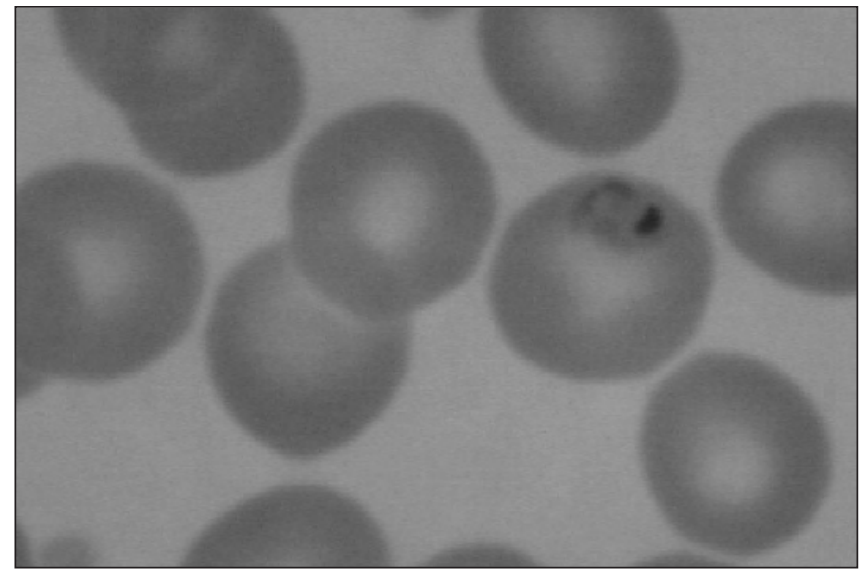

Figure 4.

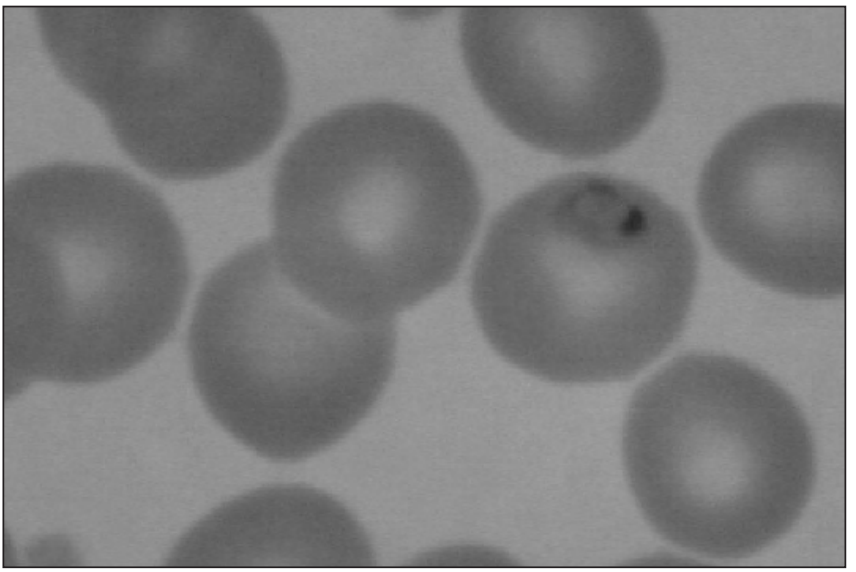

Figure 5.

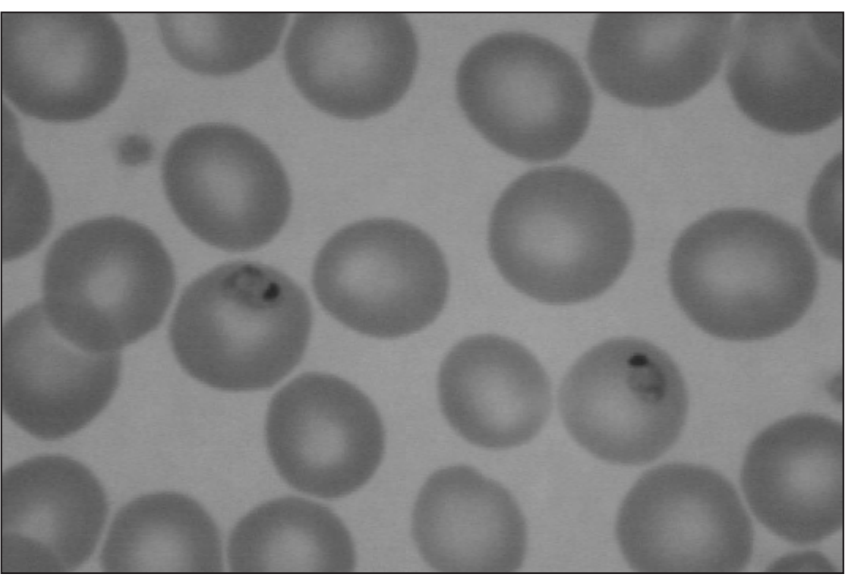

Figure 6.

\section{Discussion}

Approximately 300 to 500 million cases of malaria occur annually worldwide, particularly in children living in tropical developing countries. Infection rates have increased in recent years, secondary to factors including increased resistance of parasites to drug therapy, increased resistance of the Anopheles mosquito to insecticides, climate changes, and increased travel to endemic regions. Globally, the areas of greatest transmission include Oceania and sub-Saharan Africa; other regions with high risk of transmission, in descending order, are the Indian subcontinent, Southeast Asia, South America, and Central America. Travelers from industrialized countries are at risk for malaria as well; approximately 30,000 of them contract the disease each year. Worldwide, an alarming 700,000 to 2.7 million deaths occur each year.

Malaria transmission occurs predominantly via a bite from the female Anopheles mosquito. There are other rare means of transmission, including congenital acquisition, blood transfusion, needle sharing, and organ transplant. In the United States, Anopheles mosquitoes are present in all states, except Hawaii. Although extremely rare, mosquito-borne transmission in the US has occurred. An outbreak of malaria was reported in West Palm Beach, Florida in 2003, with 7 documented cases of Plasmodium vivax infection.

There are four species of Plasmodia: P. falciparum, P. vivax, P. ovale, and P. malariae. Human infection by each of them occurs when sporozoites are transmitted from an infected anopheline mosquito during a bite. The sporozoites travel via the bloodstream to the liver, where they invade hepatocytes and divide thousands of times into mature tissue schizonts. Each schizont contains thousands of daughter merozoites. After 6-16 days, the liver schizonts rupture, releasing merozoites into the bloodstream where they invade red blood cells.

Within the RBCs, merozoites mature from ring forms to trophozoites to mature red cell schizonts (asexual); daughter merozoites are then released and able to infect new red cells. Some merozoites will differentiate into male and female gametocytes (sexual) which circulate in the bloodstream until they are ingested by a blood-feeding anopheline mosquito. Sporozoites then form in the mosquito and will eventually reinfect humans.

Mosquito-transmitted P. vivax and P. ovale infections carry a unique risk; some parasites remain dormant in the liver and may cause late relapse by reactivating after several months.

The pathogenesis of malaria is several-fold. All four species may cause anemia via alteration of red cell membranes resulting in hemolysis, accelerated splenic clearance, and digestion of red cell proteins and hemoglobin. Additionally, red cell lysis releases tumor necrosis factor alpha, which in turn suppresses hematopoiesis. Thrombocytopenia may result from increased splenic sequestration and decreased platelet survival time.

P. falciparum may cause especially severe or fatal disease via formation of "sticky knobs" on the surface of erythrocytes. These may bind to receptors on endothelial cells in capillaries and venules, leading to sequestration and subsequent obstruction to blood flow, which can result in secondary organ dysfunction. 
Several genetic factors have been suggested as protective mechanisms against severe infection. For example, presence of the sickle cell trait has been shown to lower the risk of P. falciparum malaria, lower parasite densities, and lower rates of hospital admissions. Alpha and beta thalassemia have been shown to be associated with decreased rates of parasite multiplication, as well.

Individuals who live in endemic areas may develop partial immunity to disease after repeated infections; they become "semi-immune." This does not completely prevent infection however; after a bite from an infected mosquito, they will develop parasitemia, but the severity of symptoms may be less. This partial immunity often wanes after leaving an endemic area. Therefore, when these individuals return home to endemic areas, they must take prophylaxis.

Malaria infections usually become symptomatic during the erythrocytic stage of the parasite life cycle; incubation periods for P. falciparum are usually 12 to 14 days, 2 months for P. vivax and P. ovale, and finally 35 days for P. malariae. The erythrocytic stage of infection usually lasts approximately one to four weeks. Malarial signs and symptoms may vary, but essentially all infected patients exhibit fever; it develops with the release of merozoites from rupture red blood cells. Fevers occur in paroxysms which are often daily and irregular. Other common symptoms may include chills, sweats, headache, myalgias, fatigue, nausea, vomiting, abdominal pain, diarrhea, and cough. Signs of infection may include anemia, thrombocytopenia, splenomegaly, hepatomegaly, and jaundice. Nephrotic syndrome occurs in some cases, and is most commonly associated with P. malariae.

P. falciparum in particular has been noted to have severe morbidity and mortality. Untreated falciparum infections can be fatal. This species has the ability to invade red cells of all ages, thereby allowing high levels of parasitemia, sometimes involving more than $50 \%$ of red cells. As noted above, P. falciparum is more likely to lead to secondary end organ damage than the other species because of its ability to adhere to blood vessels and create obstruction to flow. Cerebral malaria is one rare, but potentially fatal effect of the infection. It usually presents with an impaired state of consciousness or seizures, and may result in coma or death; $20 \%$ of treated adults and $15 \%$ of children may die. Other complications of P. falciparum include oliguric renal failure, pulmonary edema/ARDS, hypoglycemia, anemia, and gastroenteritis.

Conventionally, malaria has been diagnosed by light microscopy of a Giemsa-stained thick and/or thin blood smear; this is the gold standard. The thick smear is more sensitive in the diagnosis of malaria, however the thin smear facilitates exam of the parasite morphology which aids in species identification. The thin smear is also used to quantify the percentage of parasitized red cells. PCR-based techniques are also available; they detect nucleic acid sequences specific to Plasmodium species and are helpful when light microscopy is equivocal in species identification. The sensitivity and specificity of PCR approach 100 percent. Other antigen detection methods and serologic assays are available, but their sensitivity decreases with lower levels of parasitemia.

Most patients with P. vivax, P. ovale, and P. malariae can be treated as outpatients. Those with P. falciparum however, should be admitted to the hospital for initiation of their treatment and observation for any evidence of complications. None of the available anti-malarial drugs act on all stages of the malaria life cycle to kill the parasite; therefore, patients require multi-drug regimens. Choosing an appropriate regimen depends upon the species and pattern of drug resistance.

P. ovale, P. malariae, and chloroquine-sensitive P. vivax can be treated with oral chloroquine, with cure rates exceeding 95 percent. Patients with chloroquine-resistant $\mathrm{P}$. vivax may be treated with a combination of mefloquine or quinine sulfate and doxycycline. In P. vivax and P. ovale infection, relapse after chloroquine therapy is common secondary to the presence of liver hypnozoites lying dormant. Therefore, primaquine should be given to all of these patients for fourteen days, immediately after finishing their course of chloroquine. All patients must be screened for G6PD deficiency before starting primaquine therapy to avoid hemolysis.

Chloroquine-resistant falciparum is now widespread throughout most countries; therefore, P. falciparum infections should be presumed to be chloroquine-resistant in almost all cases. The most commonly recommended regimen for this is oral quinine plus pyrimethamine-sulfadoxine or doxycycline for seven days. An alternative is quinine plus clindamycin, particularly for pregnant women or young children. Quinine-based regimens have greater than 90 percent efficacy in most parts of the world, except Southeast Asia where resistance is prevalent. There are alternative regimens as well, including mefloquine, atovaquone-proguanil, and artemisinin derivatives.

It is essential to stress the importance of taking chemoprophylaxis when patients travel to endemic areas. Most cases of malaria in the US are in patients who have not taken prophylaxis or those who have stopped taking it too soon after return home. Chloroquine should be used for areas where there is no chloroquine-resistant P. falciparum; it should be started 1-2 weeks before the patient departs and continued for 4-6 weeks after leaving the endemic area. Travelers to chloroquine-resistant areas should take mefloquine for prophylaxis. After travel to areas with P. vivax and P. ovale, patients should take primaquine during the last two weeks of a prophylaxis period.

\section{References}

1. Baird, J. Effectiveness of antimalarial drugs. New England Journal of Medicine 2005; 352: 1565.

2. Greenwood, B. Malaria. Lancet 2005; 365: 1487.

3. Kain, K. Malaria in travelers. Epidemiology, disease, and prevention. Infectious Disease Clinics of North America 1998; 12: 267.

4. Leder, K. Malaria. UpToDate version 14.2. Available at www.uptodate.com. Accessed October 2006

5. Stanley, J. Malaria. Emergency Medicine Clinics of North America 1997; 15: 113.

6. White, N. The treatment of malaria. New England Journal of Medicine 1996; 335: 800. 